\title{
Divertículos gástricos subcardiales: un hallazgo endoscópico poco frecuente
}

\section{Subcardial Gastric Diverticula: A Rare Endoscopic Finding}

Henry Royero G. ${ }^{1}$

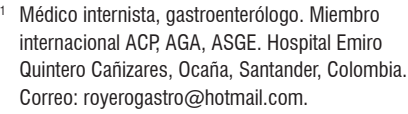

1 Médico internista, gastroenterólogo. Miembro internacional ACP, AGA, ASGE. Hospital Emiro Quintero Cañizares, Ocaña, Santander, Colombia. Correo: royerogastro@hotmail.com.

Fecha recibido: $\quad 04-04-17$ Fecha aceptado: 06-10-17

\begin{abstract}
Resumen
Los divertículos gástricos (DG) son un hallazgo infrecuente. Suelen ser diagnosticados incidentalmente por métodos radiológicos o endoscópicos; se dividen en congénitos y adquiridos; y generalmente son asintomáticos, pero su expresión clínica va desde dolor abdominal inespecífico hasta cuadros clínicos más dramáticos como perforación y sangrado. Se presenta un caso de $3 \mathrm{DG}$ en un paciente con antecedentes de colecistectomía y funduplicatura de Nissen con historia de dispepsia funcional. Su tratamiento fue conservador.
\end{abstract}

\section{Palabras clave}

Divertículo, estómago, funduplicatura de Nissen.

\section{Abstract}

Gastric diverticula are uncommon and are usually diagnosed incidentally by radiological or endoscopic methods. They are divided into congenital and acquired diverticula and are usually asymptomatic. Clinical expression ranges from nonspecific abdominal pain to more dramatic clinical conditions such as perforations and bleeding. We present a case of three gastric diverticula in a patient with a history of functional dyspepsia, cholecystectomy and Nissen fundoplication. Treatment was conservative.

\section{Keywords}

Diverticulum; stomach; Nissen fundoplication.

\section{INTRODUCCIÓN}

Los DG son dilataciones que protruyen de la pared gástrica (1); su prevalencia oscila entre $0,04 \%$ a $2,6 \%(2,3)$; y pueden ser congénitos o adquiridos (4). La mayoría de ellos son asintomáticos, pero pueden manifestarse como dispepsia, vómitos y dolor abdominal; o de formas tan severas como sangrados o perforaciones $(5,6)$. En pacientes con DG sintomáticos o complicados, la resección laparoscópica es un abordaje quirúrgico con excelentes resultados (7).

Se reporta un caso de enfermedad diverticular en el fondo gástrico, en un paciente con antecedente de funduplicatura de Nissen y colecistectomía, quien ha presentado dispepsia de largo tiempo, siendo una entidad poco frecuente y una rara asociación. Se presenta su hallazgo, además de revisar la literatura médica al respecto.

\section{DESCRIPCIÓN DEL CASO}

Un paciente masculino de 65 años consultó por dispepsia manifestada por dolor epigástrico y dificultad posprandial de varios años de evolución. Negó el uso de antiinflamatorios no esteroideos (AINE). En su historia clínica tiene una endoscopia digestiva alta (EVDA) sin lesiones sugerentes 
de divertículos 5 años antes. Sus antecedentes son funduplicatura de Nissen por reflujo gastroesofágico y hernia hiatal hace 25 años, colecistectomía por colecistitis y litiasis vesicular hace 8 años. En el examen físico el peso fue: 78 kilos, talla: 1,69 m, e índice de masa corporal (IMC): 27,36; sin signos patológicos. Previo consentimiento informado, se le practicó una EVDA (Figuras 1, 2 y 3) con los siguientes hallazgos: esófago lumen ligeramente aumentado de diámetro, en retroversión se observaron pliegues subcardiales en relación con la funduplicatura de Nissen, además de 3 orificios sacciformes entre $0,5 \mathrm{~cm}$ y $1 \mathrm{~cm}$, sin estigmas de sangrado reciente. El resto de la endoscopia sugirió gastritis crónica superficial. Las biopsias tomadas de acuerdo con el protocolo de Sidney reportaron: operative link of gastritis asessment (OLGA) I /OLGIM I. Helicobacter pylori positivo en antro y negativo en cuerpo. Se inició tratamiento para erradicación de $H$. pylori con terapia secuencial con amoxicilina, claritromicina y tinidazol por 14 días; y tratamiento sintomático de dispepsia. El paciente continuó en controles clínicos.

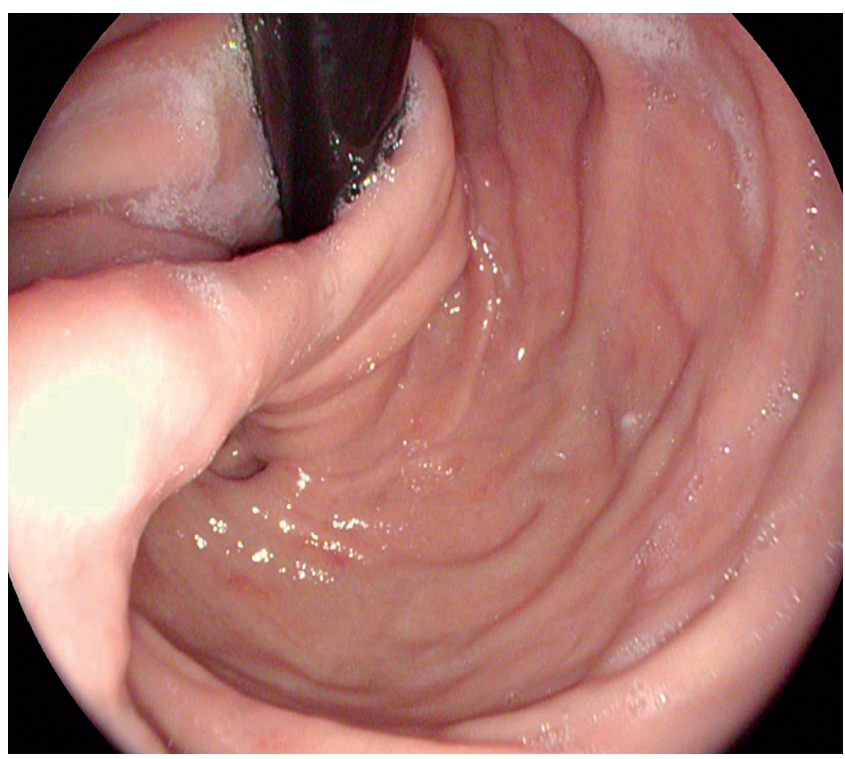

Figura 1. Vista en retroversión de pliegues subcardiales en relación con funduplicatura, además de imagen diverticular única.

\section{DISCUSIÓN}

Los DG son poco frecuentes, aunque estos varían de acuerdo con el método diagnóstico. En una gran serie de 14428 pacientes a los cuales se les indicó un estudio tomográfico toracoabdominal por diferentes motivos, se diagnosticaron incidentalmente 18 DG con una prevalencia de $0,12 \%$ (8). Una reciente revisión encontró una prevalencia de $0,04 \%$ y $0,1 \%-0,11 \%$ en estudios radiológicos con contraste y en procedimientos endoscópicos, respectivamente

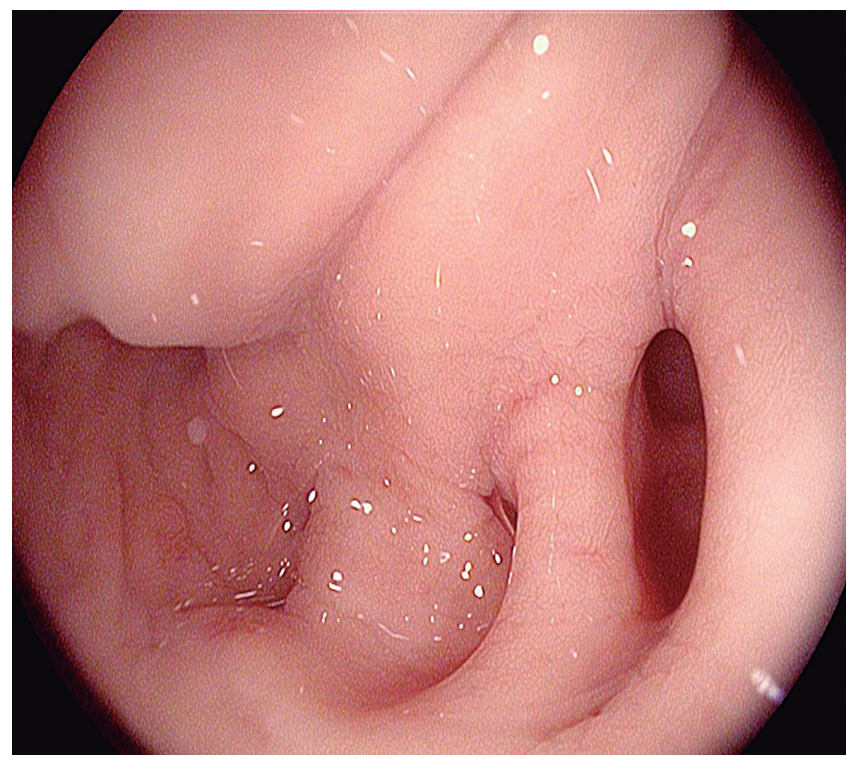

Figura 2. Acercamiento en retrovisión donde se visualizan en detalle 3 orificios sacciformes (divertículos).

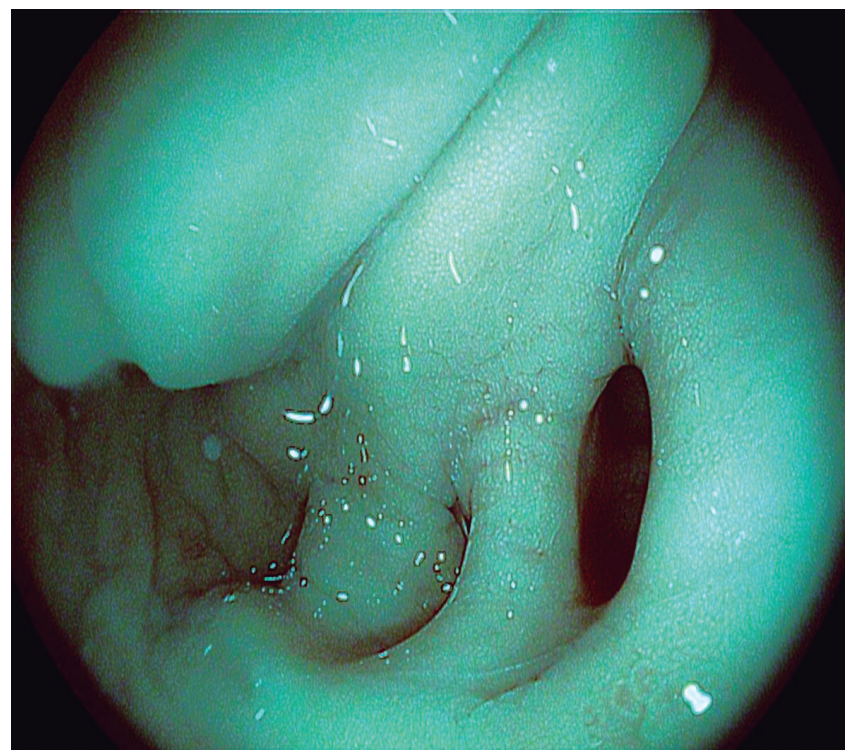

Figura 3. La misma imagen de la Figura 2 con cromoendoscopia i-SCAN.

(9). Meeroff y colaboradores (3) reportaron en autopsias una incidencia de $0,2 \%$.

Los DG pueden ser congénitos o adquiridos. Los primeros son llamados verdaderos porque comprometen todas las capas de la pared gástrica, localizándose principalmente en la pared posterior cerca de la unión gastroesofágica. En esta ubicación yuxtacardial posterior se ha encontrado una menor resistencia de la pared gástrica debido a la ausencia 
de fibras musculares longitudinales y del peritoneo; por lo tanto, cuando hay incrementos prolongados de la presión intraabdominal o intragástrica se puede condicionar la apa $\neg$ rición de un saco diverticular (10). Los segundos, conocidos como adqui $\neg$ ridos o pseudodivertículos afectan sólo la mucosa sin comprometer la capa muscular propia y se subdividen en:

- Los generados por pulsión, secundarios a condiciones asociadas con aumento de la presión intraluminal (embarazo, obstrucción pilórica, vómitos severos, tos, estreñimiento o cuerpos extraños) o al efecto erosivo de úlceras gástricas o carcinomas; y

- Los producidos por tracción, debido a adherencias perigástricas resultantes de lesiones inflamatorias del bazo, vesícula biliar, páncreas, hígado, peritoneo o cirugías del estómago. Se localizan usualmente cerca del antro gástrico $(7,9,11,12)$.

Muchos divertículos se detectan incidentalmente por medio de la tomografía axial computarizada (TAC). Se reconocen como lesiones quísticas con una pared delgada y un nivel hidroaéreo, localizándose detrás del estómago en la región paravertebral izquierda entre el bazo, la glándula suprarrenal y la crura izquierda del diafragma (8). Si un divertículo no contiene aire, puede ser malinterpretado principalmente como lesiones quísticas de la glándula suprarrenal izquierda $(12,13)$. También debe establecerse el diagnóstico diferencial con variaciones anatómicas de estructuras normales o de patologías de órganos adyacentes como bazos accesorios, tumores hepáticos, colon lleno de líquido, arterias o venas esplénicas tortuosas, masa renal exofítica en el polo superior del riñón, masas pancreáticas, aumento de grasa suprarrenal y engrosamiento de la crura diafragmática izquierda (13). Por lo anterior, se recomienda una combinación de estudios endoscópicos, radiológicos con bario y endosonográficos para corroborar el diagnóstico de un DG (14).

Varios reportes de casos informan que los DG se pueden manifestar con síntomas abdominales como vómitos y dolor abdominal $(5,15)$, o asociarse con dispepsia (16), úlcera péptica (11) y reflujo gastroesofágico $(17,18)$. También se pueden complicar con sangrados y perforación $(19,20)$.

En cuanto al manejo médico inicial, cuando están sintomáticos se recomienda el uso de antiácidos, inhibidores de la bomba de protones y una dieta blanda (21). Si los divertículos continúan con síntomas severos a pesar del tratamiento farmacológico o se complica por su gran tamaño (diámetro superior a $4 \mathrm{~cm}$ ), se indica la resolución quirúrgica por vía laparoscópica (22). Esta técnica actualmente es bien aceptada, siendo mínimamente invasiva y altamente efectiva $(23,24)$.

\section{CONCLUSIONES}

Los DG son una patología poco frecuente y suelen ser asintomáticos. En el caso descrito, y de acuerdo con la literatura médica revisada, las formaciones diverticulares subcardiales pueden estar relacionadas con cirugías previas. Además, podrían contribuir con la sintomatología descrita del tracto digestivo superior. Aunque en este paciente no se realizó ningún estudio radiológico, es importante destacar que si en una TAC se diagnostican masas adrenales izquierdas debe corroborarse o descartarse el diagnóstico de DG en el fondo gástrico, ya que es el principal diagnóstico diferencial cuando se presentan esos hallazgos imagenológicos. En los divertículos mayores de $4 \mathrm{~cm}$ se ha indicado la laparoscopia como tratamiento definitivo, debido a que tienen alta probabilidad de complicarse con perforaciones o sangrados.

\section{Fuente de apoyo financiero}

Ninguna.

\section{REFERENCIAS}

1. Podda M, Atzeni J, Messina Campanella A, Saba A, Pisanu A. Syncope with surprise: an unexpected finding of huge gastric diverticulum. Case Rep Surg. 2016;2016:1941293. https://doi.org/10.1155/2016/1941293

2. Palmer ED. Gastric diverticula. Int Abstr Surg. 1951;92(5):417-28.

3. Meeroff M, Gollán JR, Meeroff JC. Gastric diverticulum. Am J Gastroenterol. 1967;47(3):189-203.

4. Goldberg E, Raufman JP. Stomach and duodenum: anatomy and structural anomalies. En: Podolsky D, Camilleri M, Fitz JG; et al (editores). Yamada's textbook of gastroenterology. 6. a edición. Oxford: Wiley-Blackwell; 2015. pp. 60-72.

5. Mohan P, Ananthavadivelu M, Venkataraman J. Gastric diverticulum. CMAJ. 2010;182(5):E226. https://doi. org/10.1503/cmaj.090832

6. Hajini FF, Husain M, Bhat A, Bukhari SI. Gastric diverticulum a rare endoscopic finding. BMJ Case Rep. 2014 Apr 1;2014. pii: bcr2013202887.

7. Marano L, Reda G, Porfidia R, et al. Large symptomatic gastric diverticula: two case reports and a brief review of literature. World J Gastroenterol. 2013;19(36):6114-7. https:// doi.org/10.3748/wjg.v19.i36.6114

8. SchrammD,BachAG,ZipprichA, etal.Imagingfindingsofgastric diverticula. ScientificWorldJournal. 2014;2014:923098. https://doi.org/10.1155/2014/923098

9. Rashid F, Aber A, Iftikhar SY. A review on gastric diverticulum. World J Emerg Surg. 2012;7(1):1. https://doi. org/10.1186/1749-7922-7-1

10. Puente J, Aguayo J, Parilla P, et al. Divertículos gástricos. En: Balibrea Cantero JL (editor). Tratado de cirugía (vol. II): 
patología quirúrgica. 1. ${ }^{\text {a }}$ edición. Madrid: Marban Libros; 2002. pp. 2095-101.

11. Tsitsias T, Finch JG. Gastric diverticulum of the prepyloric region: a rare presentation of gastric diverticulum. Case Rep Gastroenterol. 2012;6(1):150-4. https://doi. org/10.1159/000338067

12. Zuluaga A, Ochoa J, Bustamante S, et al. Divertículos y pseudodivertículos del tracto digestivo superior: hallazgos por tomografía computarizada multidetector (TCMD): serie de casos. Rev Colomb Radiol. 2015;26(1):4139-44.

13. Feng YE, Zhang Z. Gastric diverticulum simulating a left adrenal mass: a case report and review of the literature. Oncol Lett. 2015;10(4):2477-2480. https://doi. org/10.3892/ol.2015.3559

14. Simon M, Zuber-Jerger I, Schölmerich J. True gastric diverticulum. Dig Liver Dis. 2009;41(5):370. https://doi. org/10.1016/j.dld.2008.06.016

15. Mahafza WS, Taib AA, Shahait AD, et al. Chronic gastritis in a gastric diverticulum misdiagnosed as a left adrenal mass. Indian J Surg. 2015;77(Suppl 1):150-2. https://doi. org/10.1007/s12262-015-1210-2

16. Eitzen K, Eslick GD, Daneshjoo R. Dyspepsia and gastroesophageal reflux symptoms predominate in gastric diverticulum. J Dig Dis. 2012;13(6):335-6. https://doi. org/10.1111/j.1751-2980.2012.00595.x

17. Moy BT, Marchioni Beery RM, Birk JW. Gastric diverticulum: an unusual endoscopic finding. ACG Case Rep J. 2016;3(3):150-1. https://doi.org/10.1503/cmaj.090832
18. Villazón DO, Hernández LA, Badin AV, et al. Divertículo gástrico: caso clínico y revisión de la literatura. Rev Mex Cir Endoscop. 2013;14(4):183-5.

19. Gibbons CP, Harvey L. An ulcerated gastric diverticulum-a rare cause of haematemesis and melaena. Postgrad Med J. 1984;60(708):693-5. https://doi.org/10.1136/ pgmj.60.708.693

20. Chen JH, Su WC, Chang CY, et al. Education and imaging. Gastrointestinal: bleeding gastric diverticulum. J Gastroenterol Hepatol. 2008;23(2):336.

21. Zelisko A, Rodriguez J, El-Hayek K, et al. Laparoscopic resection of symptomatic gastric diverticula. JSLS. 2014;18(1):120-4. https://doi.org/10.4293/1086808 13X13693422520648

22. Muis MO, Leitao K, Havnen J, et al. Gastric diverticulum and halitosis-a caseforsurgery? IntJSurg Case Rep.2014;5(7):4313. https://doi.org/10.1016/j.ijscr.2014.04.029

23. Tebala GD, Camperchioli I, Tognoni V, et al. Laparoscopic treatment of a gastric diverticulum. Eur Rev Med Pharmacol Sci. 2010;14(2):135-8.

24. DuBois B, Powell B, Voeller G. Gastric diverticulum: "a wayside house of ill fame” with a laparoscopic solution. JSLS. 2012;16(3):473-7. https://doi.org/10.4293/1086808 $12 X 13462882736330$ 